\title{
Correction to \\ Spectral Geometry and the Kaehler Condition for Complex Manifolds
}

\author{
Peter B. Gilkey (Princeton)
}

Inventiones math. 26, 231-258 (1974)

Professor Toru Tsujishita of the University of Tokyo has pointed out the following mistake in my above paper. He notes that on pages 241 and 253 that it is incorrectly stated that for the metric under consideration that the scalar curvature, $K$, is given by the formula:

$$
K=-g_{11 \overline{1}}-2 g_{21 \overline{1}}+g_{21} g_{2 \bar{T}}+\cdots
$$

and that the correct formula in this context is:

$$
K=-g_{11 \overline{1}}-2 g_{21 \overline{1}}+\left(\frac{1}{2}\right) g_{21} g_{2 \overline{1}}-g_{21} g_{3 / 1}+\cdots .
$$

Consequently, the statement of Lemma 3.3 should be replaced by:

$$
P_{3}(g)=2(4 \pi)^{-3} / 3\left(-2 g_{11 \overline{1}}+2 g_{21 \overline{1}}-2 g_{21} g_{2} \overline{1}+g_{21} g_{3 \overline{1}}\right) \text {. }
$$

This makes a corresponding change in the statement of Lemma 3.4. Consequently,

\begin{tabular}{|c|c|c|c|c|}
\hline 5 & $q=0$ & $q=1$ & $q=2$ & $q=3$ \\
\hline $\begin{array}{l}p=0 \\
p=1\end{array}$ & $\begin{array}{rr}2 K & +3 K_{2}+3 K_{3} \\
-6 K_{1} & +6 K_{2}+3 K_{3}\end{array}$ & $\begin{array}{r}+6 K_{1}+6 K_{2}+3 K_{3} \\
6 K+12 K_{1}+15 K_{2}+3 K_{3}\end{array}$ & $\begin{array}{l}-6 K+3 K_{2}-3 K_{3} \\
-12 K+6 K_{1}+12 K_{2}-3 K_{3}\end{array}$ & $\begin{array}{l}-4 K-6 K_{1} \quad-K_{3} \\
-6 K-12 K_{1}+3 K_{2}-3 K_{3}\end{array}$ \\
\hline $\begin{array}{l}p=2 \\
p=3\end{array}$ & $\begin{array}{l}-6 K-12 K_{1}+3 K_{2}-3 K_{3} \\
-4 K-6 K_{1}-3 K_{3}\end{array}$ & $\begin{array}{l}-12 K+6 K_{1}+12 K_{2}-3 K_{3} \\
-6 K \quad+3 K_{2}-3 K_{3}\end{array}$ & $\begin{aligned}-6 K & +12 K_{1}+15 K_{2}+3 K_{3} \\
& +6 K_{1}+6 K_{2}+3 K_{3}\end{aligned}$ & $\begin{aligned}-6 K_{1} & +6 K_{2}+3 K_{3} \\
2 K & +3 K_{2}+3 K_{3}\end{aligned}$ \\
\hline
\end{tabular}
the correct statement of Theorem 3.5 is:

Theorem 3.5. The invariants $B_{2}\left(x, D_{p, q}^{3, c}\right)$ are given by $2 / 3(4 \pi)^{-3 *}$

\begin{tabular}{|c|c|c|c|}
\hline$(p, q)$ & $q=0$ & $q=1$ & $q=2$ \\
\hline $\begin{array}{l}p=0 \\
p=1 \\
p=2\end{array}$ & $\begin{aligned} & 2 K+3 K_{2} \\
- & 2 K-6 K_{1}+3 K_{2} \\
- & 4 K-6 K_{1}\end{aligned}$ & $\begin{array}{l}-2 K+6 K_{1}+3 K_{2} \\
-4 K+12 K_{1}+6 K_{2} \\
-2 K+6 K_{1}+3 K_{2}\end{array}$ & $\begin{aligned}-4 K-6 K_{1} \\
-2 K-6 K_{1}+3 K_{2} \\
2 K+3 K_{2}\end{aligned}$ \\
\hline
\end{tabular}

This changes the correct statement of Theorem 3.7 to be:

Theorem 3.7. The invariants $B_{2}\left(x, D_{p, q}^{2, c}\right)$ are given by $(4 \pi)^{-2} / 3^{*}$ 
A slight modification in the proof of Corollary 4.3 must be made in light of the revised formulas. The remainder of the theorems in the paper are correct as stated.

Professor Tsujishita also observed that the correct statement of 3.5 gives rise to the following slightly stronger result than Corollary 4.2 :

Theorem (Tsujishita). Suppose the spectrum of the real Laplacian $\left(\frac{1}{2}\right) \triangle$ agrees with the spectrum of the complex Laplacian $\square$ on functions and 1 forms, then the metric is Kaehler.

Proof. if $n=2$, this is a consequence of Theorem 4.4. For $n=3$, these two conditions imply that:

This implies that

$$
\int_{M} 3 K_{2}+3 K_{3}=\int_{M} 12 K_{2}+6 K_{3}=0
$$

$$
\int_{M} K_{2}=0
$$

which implies that the metric is Kaehler. The case $n>3$ follows immediately from Theorem 3.6.

I would like to express my appreciation to Professor Tsujishita for pointing out the error in the paper and for suggesting the stronger version of Theorem 4.4 noted above. The example given following the proof of 4.4 shows that for $n \geqq 3$ it does not suffice to simply assume that the spectrum of the real Laplacian agrees with the spectrum of the complex Laplacian on functions alone. The last paragraph of the paper is incorrect in light of the changes made to Theorem 3.5 and should be ignored.

\author{
Peter B. Gilkey \\ Mathematics Department \\ Fine Hall \\ Princeton, N.J. 08540, USA
}

(Received February 18, 1975) 